\title{
Capacitação Tecnológica dos Laboratórios Farmacêuticos Oficiais
}

\section{Technological Capability at the Brazilian Official Pharmaceutical Laboratories}

\author{
Eduardo Rangel de Oliveira* \\ Mestre em Ciências pela EQ/UFRJ. \\ Analista da Fundação Oswaldo Cruz, Rio de Janeiro/RJ, Brasil. \\ José Vitor Bomtempo Martins \\ Doutor em Economia Industrial pela Ecole des Mines, França. \\ Professor da EQ/ UFRJ, Rio de Janeiro/RJ, Brasil. \\ Cristiane Quental \\ Doutora em Administração pela COPPEAD/UFRJ.
} Professora e Pesquisadora da Fundação Oswaldo Cruz, Rio de Janeiro/RJ, Brasil.

*Endereço: Av. Brasil, 4365, Bio-Manguinhos, Fundação Oswaldo Cruz, Rio de Janeiro/ RJ, 21040-900.E-mail: erangel@bio.fiocruz.br 


\title{
ResUMO
}

Este artigo estuda a capacitação tecnológica dos Laboratórios Farmacêuticos Oficiais [LFO] do Brasil. A análise da capacitação tecnológica pode iluminar ações próprias e governamentais para um melhor desempenho das funções básicas dos LFO, assim como para a incorporação de novas funções, em particular em gestão da inovação. O estudo inspira-se em pesquisas realizadas por Figueiredo (2000, 2003a, 2003b) e Figueiredo e Ariffin (2003) para construir uma grade de análise adaptada às peculiaridades da indústria farmacêutica e à legislação sanitária vigente. A grade permite mapear, nas diferentes dimensões da capacitação tecnológica (instalações, processos, produtos, equipamentos, capacidade organizacional e gestão do conhecimento), o grau de desenvolvimento alcançado pelos LFO (rotineiro ou inovador). Os LFO apresentam capacitação tecnológica rotineira desenvolvida especialmente em Produtos e Processos. Encontram-se diferenças significativas nas demais dimensões. Quanto às capacitações inovadoras, os LFO pouco avançaram. O principal problema de curto prazo reside na dispersão dos esforços de capacitação. Em razão da base atual e dos esforços, as perspectivas em Instalações, Processos e Capacidade Organizacional são positivas. Entretanto, o menor investimento em Produtos e Gestão do Conhecimento pode colocar em risco essas capacitações no futuro.

Palavras-chave: capacitação tecnológica; capacidade produtiva; capacidade inovadora; laboratórios farmacêuticos oficiais.

\begin{abstract}
This paper studies the technological capability in the Brazilian Official Pharmaceutical Laboratories [OPL]. The technological capability analysis could contribute to organization strategies and governmental actions in order to improve OPL basic tasks as well to incorporate new ones, particularly concerning the innovation management. Inspired in Figueiredo (2000, 2003a, 2003b) and Figueiredo and Ariffin (2003), a framework was drawn and adapted to pharmaceutical industry characteristics and current sanitary and health legislation. The framework allows to map different dimensions of the technological capability (installations, processes, products, equipments, organizational capability and knowledge management) and the level attained by OPL (ordinary or innovating capability). OPL show a good development of ordinary capabilities, particularly in Product and Processes. Concerning the other dimensions, OPL are quite diverse. In general, innovating capabilities are not much developed. In the short term, it was identified a dispersion in the capacitating efforts. Considering their present level and the absorption efforts, good perspectives can be found in Installations, Processes and Organizational Capability. A lower level of efforts in Products and Knowledge Management could undermine these capabilities in the future.
\end{abstract}

Key words: technological capability; production capability; innovative capability; pharmaceutical governmental laboratories. 


\section{INTRODUÇÃO}

Os Laboratórios Farmacêuticos Oficiais [LFO] têm importante papel na política nacional de saúde, tanto no abastecimento direto do Ministério da Saúde e das secretarias estaduais e municipais de saúde, por meio da produção de medicamentos a baixo custo, quanto na regulação do mercado farmacêutico, possibilitando com isso uma melhoria no acesso da população aos medicamentos de que necessita. Existem atualmente dezoito laboratórios filiados à Associação dos Laboratórios Oficiais do Brasil [ALFOB] (2005).

A análise das capacitações tecnológicas desses laboratórios visa a nortear ações próprias e governamentais para melhoria do desempenho de suas principais funções, assim como a incorporação de outras, como o desenvolvimento de novos medicamentos para doenças negligenciadas ${ }^{(1)}$.

Com este objetivo, buscou-se, neste artigo:

. Descrever o grau de capacitação tecnológica atual dos LFO.

. Descrever como deve se modificar essa capacitação em função dos investimentos em curso.

- Avaliar as perspectivas dos LFO, a partir da capacitação atual e dos esforços empreendidos para melhorá-la.

\section{Base Conceitual}

As capacitações organizacionais são definidas na literatura como habilidades específicas e dinâmicas da organização que "são moldadas pelas posições em ativos e pelos caminhos percorridos pela firma para criação e disseminação do conhecimento" (Alves, Bomtempo, \& Coutinho, 2005, p. 307). Envolvem a combinação de vários tipos de conhecimentos e a infra-estrutura que permite a aplicação desses conhecimentos. Nesse sentido, a literatura define capacitação tecnológica como a habilidade de fazer uso efetivo do conhecimento tecnológico (Kim, 1999, p. 111).

As capacitações são criadas e acumuladas pelos processos de aprendizagem por meio dos quais os novos conhecimentos - explícitos ou tácitos, individuais ou 
organizacionais - são adquiridos, criados e disseminados. Nesses processos, a aprendizagem individual, apesar do seu papel primordial, não é condição suficiente para a aprendizagem organizacional. Da mesma forma, a aprendizagem organizacional não é apenas o somatório das aprendizagens individuais. Somente organizações efetivas conseguem traduzir o aprendizado individual em aprendizado organizacional e, conseqüentemente, em capacitação tecnológica (Kim, 1999).

Nonaka e Takeuchi (1997) descrevem o processo de criação do conhecimento organizacional como processo contínuo, incremental e em espiral de criação e disseminação do conhecimento, através da conversão do conhecimento tácito em tácito (socialização), do conhecimento tácito em explícito (externalização), do conhecimento explícito em tácito (internalização) e do conhecimento explícito em explícito (combinação) (p. 5).

No processo de aprendizagem deve ser ainda destacado o papel crucial da capacidade de absorção. Esta não é somente a aquisição e assimilação do conhecimento por parte da organização, mas a habilidade para explorá-lo (Cohen \& Levinthal, 1990, p. 128). Segundo os autores, a capacidade de absorção é função da base de conhecimento existente e dos esforços de internalização dos novos conhecimentos, a partir da resolução interativa de problemas internos. Articulando essas duas dimensões, Kim (1999) propôs a tabela a seguir (Tabela 1), que será utilizado como ferramenta na análise da dinâmica do aprendizado tecnológico neste artigo.

\section{Tabela 1: Capacidade de Absorção}

\section{Intensidade de Esforços}

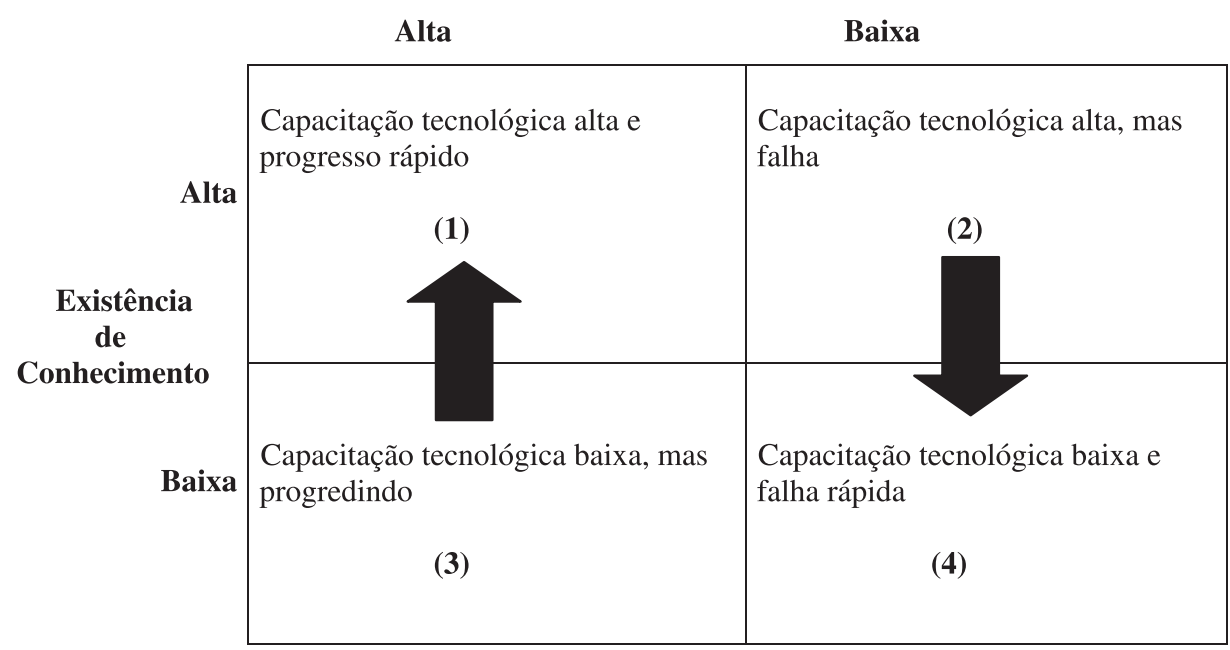

Fonte: Kim (1999, p. 115). 
A proposição de Kim (1999) mostra que, mesmo um nível elevado de conhecimento não assegura o progresso tecnológico. Caso não sejam despendidos os esforços suficientes (quadrante 2) para que este seja absorvido pela empresa, ao invés de se caminhar para o quadrante 1 (objetivo principal), a tendência é que se caminhe para o quadrante 4 , já que a dinâmica existente no processo de absorção do conhecimento tornará o conhecimento existente obsoleto. De maneira contrária, quando se está no quadrante 3, mesmo partindo de uma base de conhecimento limitada, a tendência é que, devido aos esforços empreendidos, a organização possa chegar ao quadrante 1 .

\section{Metodologia}

\section{A Escolha dos Laboratórios Analisados}

Para a análise da capacitação tecnológica dos laboratórios farmacêuticos oficiais brasileiros foi realizada uma pesquisa de campo junto a quatro dos dezoito laboratórios oficiais filiados a ALFOB (2005). Devido à grande concentração de capacidade instalada, a seleção dos laboratórios para a pesquisa levou em conta a capacidade produtiva dos laboratórios, selecionando grandes laboratórios. O atendimento à legislação sanitária brasileira também foi levado em conta para a escolha. Deu-se prioridade aos laboratórios que têm ou estão em vias de obter o certificado de boas práticas de fabricação, de acordo com os dados da Agência Nacional de Vigilância Sanitária [ANVISA] (Recuperado em 20 novembro, 2004, de http://www.anvisa.gov.br).

\section{A Grade de Análise Aplicada}

A grade de análise utilizada para a descrição da capacitação tecnológica dos LFO deriva de modelo desenvolvido originalmente por Lall (1992), e Bell e Pavitt (1995), adaptado por Figueiredo (2003b) para a indústria siderúrgica.

Neste modelo, a competência tecnológica é analisada ao longo das funções tecnológicas da empresa ${ }^{(2)}$ associadas ao seu fluxo produtivo, sendo categorizada segundo níveis de dificuldade. Este modelo sugere que a acumulação se processa das categorias mais simples para as mais complexas. Identifica-se um conjunto básico de funções em cada categoria principal, que deve ser incorporado pela empresa, a fim de garantir uma operação comercial bem sucedida. Esse conjunto básico deverá ampliar-se à medida que a empresa for assumindo tarefas mais complexas. 
O modelo distingue ainda, para cada função tecnológica, dois níveis de capacitação: a rotineira e a inovadora. A capacitação rotineira diz respeito às atividades realizadas em um determinado patamar de eficiência: são as funções tecnológicas necessárias para usar a tecnologia, o conhecimento e os mecanismos organizacionais. A capacitação inovadora, por sua vez, é aquela que permite criar, modificar ou aperfeiçoar produtos e processos.

A adaptação do modelo de Figueiredo (2000, 2003a, 2003b) e Figueiredo e Ariffin (2003) para a análise da capacitação tecnológica dos LFO resultou na grade de análise descrita na Tabela 2, a seguir. Segundo a grade de análise desenvolvida para este artigo, o nível de capacitação tecnológica dos LFO deve ser avaliado segundo funções relacionadas às seguintes variáveis: Instalações, Processos, Produtos, Equipamentos, Capacidade Organizacional e Gestão do Conhecimento. Cada uma dessas variáveis deve ser estudada segundo níveis de capacitação Rotineira e Inovadora, considerando-se níveis básicos, intermediários e avançados em cada caso.

A construção desta grade de análise foi baseada em estudos existentes sobre a indústria farmacêutica, na legislação sanitária existente ( $\left.R D C n^{\circ} 210 / 2003\right)$ e nos processos de validação preconizados por esta legislação, assim como pelas legislações internacionais. Foram levados em conta também os fatores envolvidos no desenvolvimento da capacitação tecnológica preconizados na literatura. Além disso, a pesquisa valeu-se do conhecimento tácito de um dos autores, adquirido durante nove anos de trabalho ligado diretamente à área produtiva em laboratórios farmacêuticos oficiais. O modelo foi validado por três especialistas na área, pessoas com larga experiência industrial e com atuação junto aos laboratórios públicos. 


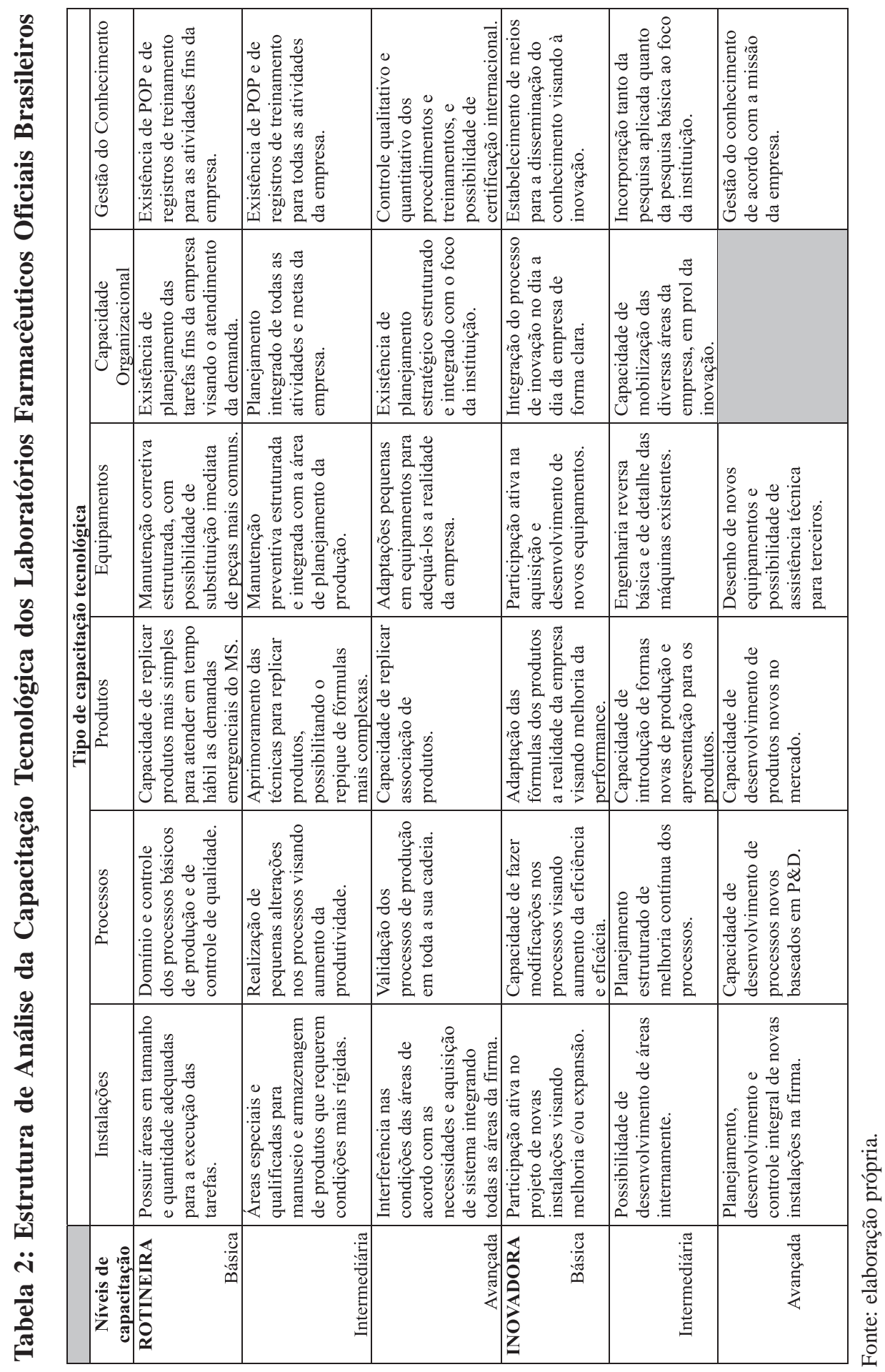




\section{Levantamento dos Dados}

A pesquisa de campo junto aos quatro laboratórios farmacêuticos oficiais foi realizada entre novembro de 2004 e abril de 2005.

Cada visita durou entre oito e dezesseis horas. Foram feitas entrevistas baseadas em um questionário semi-estruturado, elaborado a partir do quadro de análise desenvolvido, com pessoas de diversos níveis da empresa, passando pela diretoria, gerência, supervisão, chegando até o nível operacional. Ao lado das entrevistas, foram feitas também observações durante o dia-a-dia de funcionamento das organizações nas áreas de produção, controle de qualidade, garantia da qualidade, almoxarifado, manutenção, pesquisa e desenvolvimento e de recursos humanos, onde ocorreram entrevistas com os gerentes de área, supervisores e com o pessoal operacional. Essas observações serviram de complemento e confirmação dos resultados obtidos nas entrevistas. Consideramse ainda a documentação e os registros de arquivo que puderam ser consultados para a complementação e comparação das informações recebidas. Buscou-se utilizar um conjunto de informações complementares que pudessem corroborar os resultados das entrevistas de forma a possibilitar o preenchimento da grade de análise.

\section{REsultados}

\section{Descrição da Capacitação Tecnológica dos LFO}

São apresentados a seguir os graus de capacitação tecnológica encontrados em cada uma das dimensões analisadas e as possibilidades de progresso que podem ser alcançadas pelos laboratórios, em função dos investimentos que estão em curso.

\section{Instalações}

A Tabela 3 apresenta o nível de capacitação tecnológica em que estão os laboratórios oficiais em relação a instalações, e a tendência futura de acordo com a visão deste artigo. 
Tabela 3: Capacitação Tecnológica dos Laboratórios Oficiais em Relação a Instalações

\begin{tabular}{|c|c|c|c|c|}
\hline \multicolumn{5}{|c|}{ Capacitação Tecnológica em Instalações } \\
\hline $\begin{array}{c}\text { Níveis de } \\
\text { capacitação }\end{array}$ & Laboratório A & Laboratório B & Laboratório C & Laboratório D \\
\hline \multirow{2}{*}{\multicolumn{5}{|c|}{$\begin{array}{r}\text { ROTINEIRA } \\
\text { Básica }\end{array}$}} \\
\hline & & & & \\
\hline \multicolumn{5}{|l|}{ Intermediária } \\
\hline \multicolumn{5}{|l|}{ Avançada } \\
\hline \multirow{2}{*}{\multicolumn{5}{|c|}{$\begin{array}{r}\text { INOVADORA } \\
\text { Básica }\end{array}$}} \\
\hline & & & & \\
\hline \multicolumn{5}{|l|}{ Intermediária } \\
\hline Avançada & & & & \\
\hline
\end{tabular}

Fonte: elaboração própria.

Grau de capacitação atual.

Grau de capacitação que pode ser alcançado.

A dimensão Instalações foi a que se mostrou mais homogênea em relação à capacitação tecnológica dos LFO. Todos os laboratórios estudados têm capacitação tecnológica nos três níveis rotineiros analisados, porém só os cumprem de forma parcial, em virtude principalmente da existência de gargalos de produção, e falta de controle total sobre as condições das suas áreas. O laboratório $\mathbf{A}$ ainda apresenta também características do nível inovador básico, já que foi o único que demonstrou alguma participação dos funcionários envolvidos nos projetos para novas instalações.

Os investimentos ora realizados nos laboratórios estudados; entretanto devem capacitá-los a cumprir integralmente os níveis rotineiros de capacitação. Quanto aos níveis inovadores, as instituições estudadas não demonstraram interesse em alcançá-los, pelo menos no médio prazo, por entenderem que essas características não fazem parte da missão dos laboratórios.

\section{Processos}

A Tabela 4 representa o nível de capacitação tecnológica em que se encontram os laboratórios oficiais em processos. 


\section{Tabela 4: Capacitação Tecnológica dos Laboratórios Oficiais em Relação a Processos}

\begin{tabular}{|r|c|c|c|c|}
\hline \multicolumn{5}{|c|}{ Capacitação Tecnológica em Processos } \\
\hline $\begin{array}{r}\text { Níveis de } \\
\text { capacitação }\end{array}$ & Laboratório A & Laboratório B & Laboratório C & Laboratório D \\
\hline $\begin{array}{r}\text { ROTINEIRA } \\
\text { Básica }\end{array}$ & & & & \\
\hline Intermediária & & & & \\
\cline { 2 - 5 } Avançada & & & & \\
\hline $\begin{array}{r}\text { INOVADORA } \\
\text { Básica }\end{array}$ & & & & \\
\hline \multirow{2}{*}{$\begin{aligned} \text { Intermediária } \\
\text { Avançada }\end{aligned}$} & & & & \\
\hline & & & & \\
\hline
\end{tabular}

Fonte: elaboração própria.

Grau de capacitação atual. Grau de capacitação que pode ser alcançado.

$\mathrm{Na}$ análise da dimensão Processos surgiram algumas diferenças significativas, sobretudo em relação ao processo de construção da capacitação tecnológica. Alguns laboratórios vêm trabalhando de forma mais consistente e outros de forma descontínua. Por isso, encontram-se características pertencentes a quase todos os níveis de capacitação. Mesmo assim, o nível mais avançado de capacitação tecnológica, atingido de maneira uniforme, foi encontrado nos laboratórios $\mathbf{A}$ e $\mathbf{B}$. O nível rotineiro intermediário foi atingido de forma incompleta, apesar de ambos apresentarem características inerentes a outros níveis superiores. Quanto às outras instituições, o comportamento é semelhante, tendo como única diferença o fato de o laboratório $\mathbf{C}$ apresentar mais dificuldades no nível rotineiro básico de capacitação de acordo com o nosso estudo, embora apresente características até do nível inovador avançado.

Nos próximos anos, caso as instituições tenham sucesso na conclusão do processo de validação em curso, o nível rotineiro avançado deve ser alcançado, de maneira uniforme, por todos os laboratórios analisados. Em relação aos níveis inovadores, parece haver necessidade de maiores investimentos e melhoria no planejamento das ações internamente.

\section{Produtos}

A Tabela 5 representa o nível de capacitação tecnológica em que se encontram os laboratórios oficiais no que diz respeito a produtos. 
Tabela 5: Capacitação Tecnológica dos Laboratórios Oficiais em Relação a Produtos

\begin{tabular}{|c|c|c|c|c|}
\hline \multicolumn{5}{|c|}{ Capacitação Tecnológica em Produtos } \\
\hline $\begin{array}{c}\text { Níveis de } \\
\text { capacitação } \\
\text { tecnológica }\end{array}$ & Laboratório A & Laboratório B & Laboratório C & Laboratório D \\
\hline $\begin{array}{r}\text { ROTINEIRA } \\
\text { Básica }\end{array}$ & & & & \\
\hline Intermediária & & & & \\
\hline Avançada & & & & \\
\hline $\begin{array}{r}\text { INOVADORA } \\
\text { Básica }\end{array}$ & & & & \\
\hline Intermediária & & & & \\
\hline Avançada & & & & \\
\hline
\end{tabular}

Fonte: elaboração própria.

Grau de capacitação atual.

Esta foi a dimensão da capacitação tecnológica em que os laboratórios farmacêuticos oficiais apresentaram o nível mais elevado de maneira uniforme. Dois laboratórios, $\mathbf{B}$ e $\mathbf{C}$, alcançam o nível rotineiro avançado e dois, A e D, o nível rotineiro intermediário de maneira parcial. No caso dos dois últimos, o número de produtos mais complexos e de associações ainda é pequeno.

Na dimensão Produtos, a situação dos laboratórios $\mathbf{A}$ e $\mathbf{D}$ é muito próxima em relação à capacitação tecnológica, até mesmo no comportamento irregular (o maior encontrado nas dimensões estudadas) em relação às perspectivas em função dos investimentos em curso. Da mesma forma, o comportamento dos outros dois laboratórios também apresenta muitas semelhanças, apenas diferindo com relação ao desenvolvimento de capacitação inovadora no nível mais avançado apresentada pelo laboratório $\mathbf{C}$. Não foi identificada capacitação inovadora em $\mathbf{B}$. Ao contrário de $\mathbf{C}, \mathbf{B}$ não tem características inovadoras. $\mathbf{O}$ laboratório $\mathbf{C}$ tende a conseguir cumprir parcialmente o nível inovador avançado devido aos esforços que têm sido feitos para melhoria geral de sua performance, e uma melhor integração das atividades em relação aos outros laboratórios estudados.

\section{Equipamentos}

A Tabela 6 mostra o nível de capacitação tecnológica encontrado nos laboratórios oficiais no que diz respeito a equipamentos. 


\section{Tabela 6: Capacitação Tecnológica dos Laboratórios Oficiais em Relação a Equipamentos}

\begin{tabular}{|c|c|c|c|c|}
\hline \multicolumn{5}{|c|}{ Capacitação Tecnológica em Equipamentos } \\
\hline $\begin{array}{c}\text { Níveis de } \\
\text { capacitação } \\
\text { tecnológica }\end{array}$ & Laboratório A & Laboratório B & Laboratório C & Laboratório D \\
\hline $\begin{array}{c}\text { ROTINEIRA } \\
\text { Básica }\end{array}$ & & & & \\
\hline Intermediária & & & & \\
\hline Avançada & & & & \\
\hline $\begin{array}{r}\text { INOVADORA } \\
\text { Básica }\end{array}$ & & & & \\
\hline Intermediária & & & & \\
\hline Avançada & & & & \\
\hline
\end{tabular}

Fonte: elaboração própria.

Grau de capacitação atual. Grau de capacitação que pode ser alcançado.

Em equipamentos foi encontrado o maior contraste em relação à capacitação tecnológica, ao se aplicar a grade de análise. Três dos quatro laboratórios analisados (A, C e D) apresentam comportamento bem parecido, tendo dificuldades de cumprimento do nível rotineiro básico, devido à falta de um controle de estoque de peças de reposição para os seus equipamentos, e ausência de procedimentos tanto para manutenção preventiva quanto para manutenção corretiva. Nos laboratórios A e D, aliás, estes processos são tratados separadamente, sendo a manutenção preventiva controlada pela produção. Esses laboratórios, diferentemente de $\mathbf{C}$, não apresentaram ações que indiquem a obtenção de progressos em equipamentos.

O laboratório B, contudo, situa-se no nível rotineiro avançado, e com amplas possibilidades de obter um nível mais elevado em curto espaço de tempo, em virtude da boa estrutura de manutenção montada, respaldada por procedimentos escritos, um software, que controla os processos de manutenção, e a existência de controle de estoque, que abrange mais de $50 \%$ das peças de reposição dos equipamentos.

\section{Capacidade Organizacional}

A Tabela 7 ilustra o grau de capacitação tecnológica existente nos laboratórios oficiais e a tendência futura. 


\section{Tabela 7: Capacitação Tecnológica dos Laboratórios Oficiais em Relação à Capacidade Organizacional}

\begin{tabular}{|c|c|c|c|c|}
\hline \multicolumn{5}{|c|}{ Capacitação Tecnológica em Capacidade Organizacional } \\
\hline $\begin{array}{l}\text { Níveis de } \\
\text { capacitação } \\
\text { tecnológica }\end{array}$ & Laboratório A & Laboratório B & Laboratório C & Laboratório D \\
\hline $\begin{array}{l}\text { ROTINEIRA } \\
\text { Básica }\end{array}$ & & & & \\
\hline Intermediária & & & & \\
\hline Avançada & & & & \\
\hline $\begin{array}{r}\text { INOVADORA } \\
\text { Básica }\end{array}$ & & & & \\
\hline Intermediária & & & & \\
\hline Avançada & & & & \\
\hline
\end{tabular}

Fonte: elaboração própria.

Grau de capacitação atual.

Grau de capacitação que pode ser alcançado.

Esta dimensão da capacitação tecnológica foi aquela em que se obteve menor descontinuidade, porém menor capacitação acumulada e nenhuma característica inovadora nos laboratórios analisados.

Todos os laboratórios possuem planejamento de produção, mas segundo horizontes de tempo muito diferentes. Os laboratórios diferem ainda na capacidade de cumprir efetivamente os planos de produção. Na organização A o planejamento engloba apenas um mês, em $\mathbf{B}$ um ano, na organização $\mathbf{C}$ apenas uma semana, e em $\mathbf{D}$ seis meses. Assim, pode-se afirmar que o laboratório A, e principalmente o laboratório $\mathbf{C}$, possuem muitas dificuldades para o cumprimento de seu planejamento de produção, devido ao pequeno tempo contemplado por este. Nenhuma das instituições apresentou planejamento estruturado para a sua área de Pesquisa e Desenvolvimento [P\&D], e apenas o laboratório A apresentou um planejamento estratégico, contemplando apenas um ano. As instituições B e D apresentaram apenas projetos de planejamento estratégico, que devem abranger cinco anos, e o laboratório $\mathbf{C}$ afirmou ainda estar estudando esta possibilidade.

Em virtude das informações sobre investimentos em curso, apenas os laboratórios B e D devem apresentar progressos em capacidade organizacional, de acordo com o modelo sugerido. Em contrapartida, o laboratório $\mathbf{C}$ pode comprometer toda a sua capacitação tecnológica nas demais dimensões, devido ao baixo grau de capacidade organizacional encontrado e à carência de investimentos nessa 
área. Destaque-se a grande influência da capacidade organizacional em relação às outras dimensões estudadas.

\section{Gestão do Conhecimento}

A Tabela 8 representa o nível de capacitação tecnológica em que se encontram os laboratórios oficiais no que diz respeito à gestão do conhecimento.

\section{Tabela 8: Capacitação Tecnológica dos Laboratórios Oficiais em Relação à Gestão do Conhecimento}

\begin{tabular}{|c|c|c|c|c|}
\hline \multicolumn{5}{|c|}{ Capacitação Tecnológica em Gestão do Conhecimento } \\
\hline $\begin{array}{c}\text { Níveis de } \\
\text { capacitação } \\
\text { tecnológica }\end{array}$ & Laboratório A & Laboratório B & Laboratório C & Laboratório D \\
\hline $\begin{array}{l}\text { ROTINEIRA } \\
\text { Básica }\end{array}$ & & & & \\
\hline Intermediária & & & & \\
\hline Avançada & & & & \\
\hline $\begin{array}{l}\text { INOVADORA } \\
\text { Básica }\end{array}$ & & & & \\
\hline Intermediária & & & & \\
\hline Avançada & & & & \\
\hline
\end{tabular}

Fonte: elaboração própria.

Grau de capacitação atual. Grau de capacitação que pode ser alcançado.

Em gestão do conhecimento, os dados encontrados mostram que todos os laboratórios analisados conseguem cumprir totalmente o nível rotineiro básico da estrutura de capacitação tecnológica sugerida, pois possuem estruturas pautadas em Procedimentos Operacionais Padronizados [POP] para as sua tarefas fins. Apresentam porém carências de padronização nas áreas administrativas e de pesquisa (caso dos laboratórios $\mathbf{A}, \mathbf{C}$ e $\mathbf{D}$, já que $\mathbf{B}$ não tem $\mathbf{P} \& D$ ). Muito provavelmente por causa disso o laboratório $\mathbf{B}$ encontra-se um pouco acima dos demais nessa dimensão.

As instituições analisadas carecem também de maior controle sobre os treinamentos, assim como de mecanismos para a disseminação do conhecimento. Em razão disso, a perspectiva de algum progresso pôde ser identificada apenas para os laboratórios B e C. No caso do primeiro, em função do certificado internacional emitido pela Organização Pan-Americana de Saúde [OPAS] e do projeto de controle dos treinamentos. No caso de $\mathbf{C}$, existe a intenção de 
padronização de todas as atividades da instituição através da implantação de um sistema de gestão integrada.

\section{Resumindo}

Os laboratórios apresentam razoável capacidade rotineira, especialmente no que diz respeito a Produtos e Processos. Com os investimentos em curso alguns devem atingir níveis de capacitação mais avançados.

O mesmo deve ocorrer com as Instalações. Apesar de ser atualmente um dos principais gargalos enfrentados pelos laboratórios (o que se apresenta na análise como descontinuidade no processo de acumulação de capacitação, com dispersão de esforços em várias direções), as obras em curso devem minimizar os problemas enfrentados.

Nas outras dimensões, o comportamento dos laboratórios é mais variado. Destaca-se o Laboratório B não só pelo nível de capacitação alcançado, mas pela consistência no processo de acumulação de capacitação.

No que diz respeito à capacitação inovadora, os LFO pouco avançaram. Os Laboratórios A, C e D apresentam padrões bastante semelhantes, decorrentes de suas atividades de P\&D. O Laboratório B, voltado para a excelência produtiva, está realizando investimentos que podem levá-lo ao nível mais alto de capacitação tecnológica inovadora em Equipamentos, porém carece de investimentos para alcançar os níveis inovadores em Processos, Produtos e Gestão do Conhecimento.

\section{Visão Dinâmica do Processo de Capacitação Tecnológica dos LFO}

$\mathrm{Na}$ análise dos resultados do artigo procurou-se obter uma visão dinâmica do processo de capacitação tecnológica dos LFO. Para isso, utilizou-se como base a proposta feita por Kim (1999) que relaciona a capacidade de absorção do conhecimento à base de conhecimento existente nas organizações e à intensidade de esforços para internalizá-la e disseminá-la na instituição (p. 115). Esse modelo foi adaptado para o estudo dos laboratórios oficiais, trocando-se a existência de conhecimento pelo grau de capacitação tecnológica acumulada pelos laboratórios. Seguem abaixo os resultados obtidos.

\section{O LaboratórioA}

As evidências mostram que na maioria das dimensões da capacitação tecnológica, apesar de o grau de capacitação ser baixo, o laboratório tem empreendido esforços para aumentá-la, especialmente em Processos, Instalações e Capacidade Organizacional, com boas perspectivas para o seu desenvolvimento futuro. 
No caso de Equipamentos, justamente a área mais carente de capacitação, o laboratório A não está investindo e se encontra no nível mais baixo de capacidade de absorção.

Em relação a Produtos e Gestão do Conhecimento, constata-se que, apesar de a capacitação existir, a intensidade de esforços em relação a essas duas dimensões tem sido pequena, ou direcionada de maneira equivocada. Como consequiência, caso o laboratório não reveja sua visão a esse respeito, existe o risco de que o conhecimento existente se perca ou se torne obsoleto, prejudicando o progresso da organização.

A Tabela 9 representa a capacidade de absorção encontrada no laboratório $\mathbf{A}$ em relação aos tipos de capacitação tecnológica estudados.

\section{Tabela 9: Capacidade de Absorção do Laboratório A}

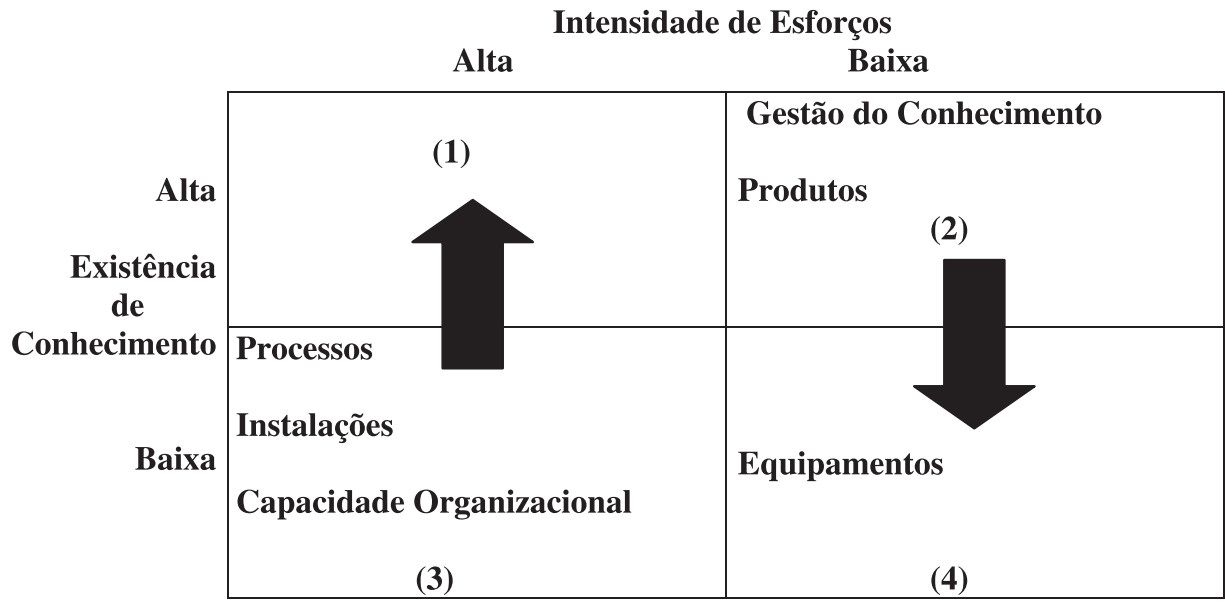

(1) Capacitação tecnológica alta e progresso rápido.

(2) Capacitação tecnológica alta, mas com risco de obsolescência.

(3) Capacitação tecnológica baixa, mas progredindo.

(4) Capacitação tecnológica baixa e obsolescência rápida.

Fonte: elaboração própria a partir de $\operatorname{Kim}(1999$, p. 115).

\section{O Laboratório B}

A análise dos dados coletados torna evidente a intenção do laboratório $\mathbf{B}$ de não direcionar seus esforços para a inovação, a não ser em Equipamentos, onde há capacitação acumulada e grande intensidade de esforços, gerando excelentes perspectivas para o futuro. 
Em Processos e Produtos, apesar de existir capacitação, a intensidade de esforços é baixa, em razão da intenção de não investir em P\&D.

Nas outras três dimensões constata-se baixo grau de capacitação. Quanto à intensidade de esforços, ela existe em Instalações, e Capacidade Organizacional, mas não em Gestão do Conhecimento, pelo que foi observado.

A Tabela 10 mostra a capacidade de absorção encontrada no laboratório $\mathbf{B}$, a partir das observações da pesquisa.

Tabela 10: Capacidade de Absorção do Laboratório B

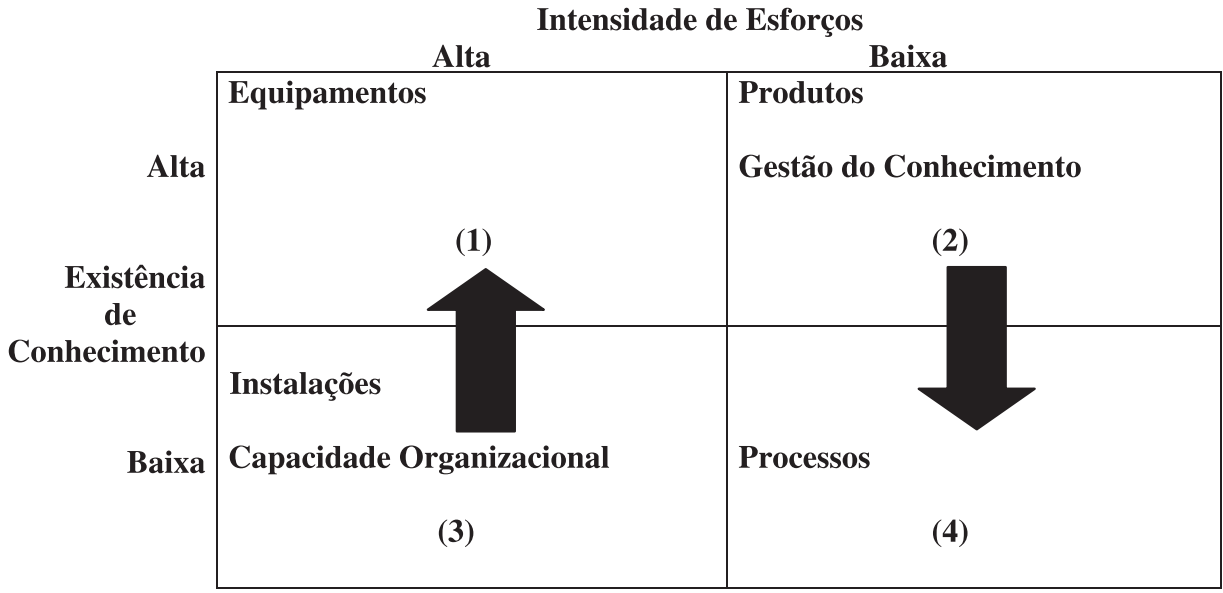

(1) Capacitação tecnológica alta e progresso rápido.

(2) Capacitação tecnológica alta, mas com risco de obsolescência.

(3) Capacitação tecnológica baixa, mas progredindo.

(4) Capacitação tecnológica baixa e obsolescência rápida.

Fonte: elaboração própria a partir de $\operatorname{Kim}(1999$, p. 115).

\section{O Laboratório C}

A Tabela 11 representa a capacidade de absorção encontrada no laboratório $\mathbf{C}$, em relação aos tipos de capacitação tecnológica estudados. 


\section{Tabela 11: Capacidade de Absorção do Laboratório C}

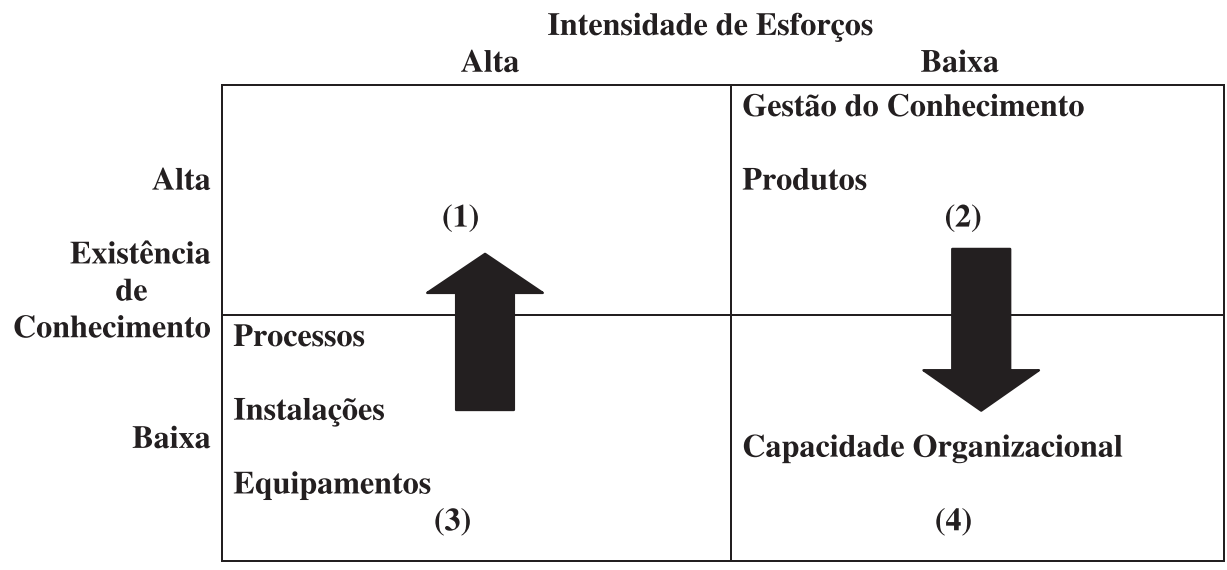

(1) Capacitação tecnológica alta e progresso rápido.

(2) Capacitação tecnológica alta, mas com risco de obsolescência.

(3) Capacitação tecnológica baixa, mas progredindo.

(4) Capacitação tecnológica baixa e obsolescência rápida.

Fonte: elaboração própria a partir de $\operatorname{Kim}(1999$, p. 115).

A pesquisa realizada na organização $\mathbf{C}$ mostra que em quase todas as dimensões da capacitação tecnológica o laboratório $\mathbf{C}$ tentou pular etapas. Como conseqüência, o que se observa é um comportamento irregular em relação à capacidade de absorção. A única dimensão em que não há este desequilíbrio é a Capacidade Organizacional, justamente a área mais carente de capacidade de absorção. Nas outras dimensões, a instituição apresenta grande desequilíbrio, exibindo um nível baixo de capacitação tecnológica acumulada em três delas, mas grande intensidade de esforços, caso de Processos, Instalações e Equipamentos, e existência de conhecimento e deficiência de esforços em Gestão do Conhecimento e Produtos.

\section{O Laboratório D}

Em três dimensões, o laboratório $\mathbf{D}$ apresentou baixo nível de capacitação acumulada, mas grandes esforços para aumentá-lo: Instalações, Processos, e Capacidade Organizacional. Em Produtos e Gestão do Conhecimento foi identificada a existência de capacitação, mas insuficiência de esforços. Já em Equipamentos, não foi possível identificar a existência de capacitação nem de esforços. A Tabela 12 representa a capacidade de absorção encontrada no laboratório $\mathbf{D}$ em relação aos tipos de capacitação tecnológica. 
Tabela 12: Capacidade de Absorção do Laboratório D

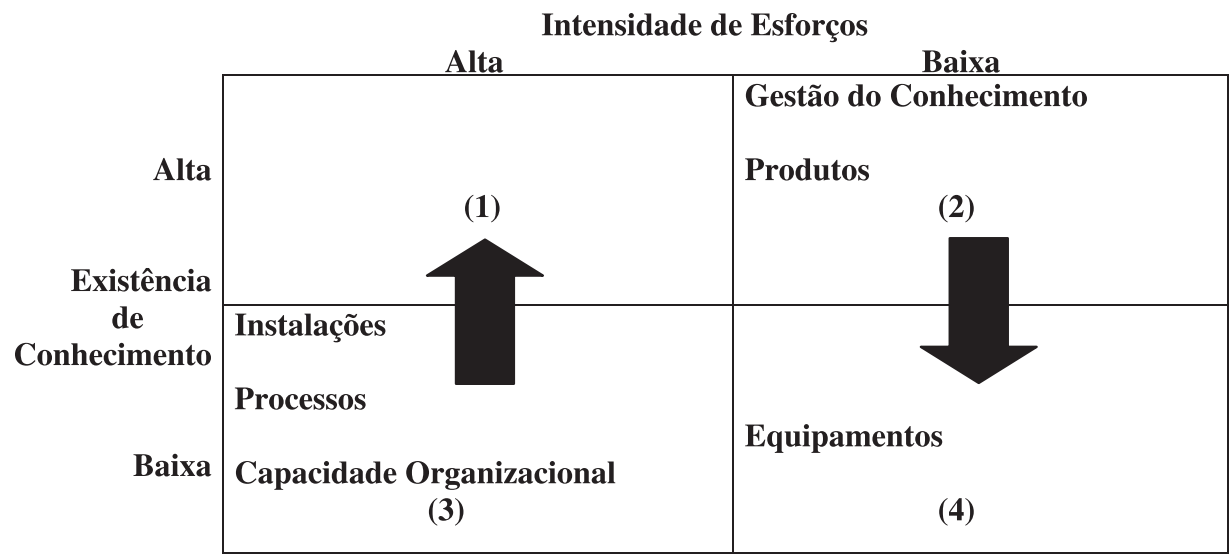

(1) Capacitação tecnológica alta e progresso rápido.

(2) Capacitação tecnológica alta, mas com risco de obsolescência.

(3) Capacitação tecnológica baixa, mas progredindo.

(4) Capacitação tecnológica baixa e obsolescência rápida.

Fonte: elaboração própria a partir de $\operatorname{Kim}(1999$, p. 115).

\section{Resumindo}

Numa visão de longo prazo, ganham grande importância os esforços realizados para incorporar novos conhecimentos à organização. Nesse sentido, embora a capacitação em Instalações, Processos e Capacidade Organizacional não seja hoje tão alta, os investimentos realizados em quase todos os laboratórios analisados apontam essas dimensões com boas perspectivas para o futuro. Por outro lado, embora a capacitação em Produtos e Gestão do Conhecimento seja hoje razoável, tende a se tornar obsoleta pelo baixo investimento institucional e/ou pelo direcionamento discutível dos laboratórios nessas duas dimensões.

\section{ConSiderações Finais}

Retomando os objetivos deste artigo, as considerações finais tecidas vão no sentido de contribuir para um processo de capacitação tecnológica dos laboratórios farmacêuticos oficiais, sólido e consistente, de forma a possibilitar o melhor cumprimento de sua missão no curto e no longo prazo. O levantamento da capacitação tecnológica dos quatro principais laboratórios farmacêuticos oficiais, assim como da perspectiva de evolução dessa capacitação, permite que sejam 
alinhadas recomendações em três pontos principais: a dispersão de esforços, os investimentos de curto prazo e os investimentos de longo prazo.

a) A dispersão de esforços ao longo de vários níveis de capacitação e sua aplicação a apenas alguns segmentos das organizações fazem com que não se obtenham os benefícios esperados. Nesse sentido, uma primeira recomendação seria no sentido de evitar este tipo de descontinuidade no processo de acumulação de capacitação. A superação dessas descontinuidades pode render frutos mais que proporcionais.

b) Considerando os investimentos atuais na acumulação de capacitações tecnológicas, parece que, em curto prazo, os laboratórios cumprirão de forma integral os níveis básico, intermediário e avançado de capacitação rotineira nas dimensões Produto, Processo e Instalações. Nas demais, investimentos se fazem necessários.

Destaca-se aqui a necessidade de investimento em Capacidade Organizacional, especialmente em planejamento, pelo seu nível bastante reduzido; e em Gestão do Conhecimento, apresentada nas entrevistas como grande preocupação organizacional, mas que não se transforma em ações na prática. Nesse caso, o investimento deve basear-se não apenas na aquisição de conhecimento individual, mas principalmente na sua transformação em conhecimento organizacional.

c) Considerando o longo prazo, permanece a necessidade de investimento em Gestão do Conhecimento, e aparece também a necessidade de investimento em Produtos. A falta de investimento nessas dimensões pode tornar obsoleta a capacitação já conseguida.

Dada a configuração da capacitação tecnológica dos laboratórios estudados, pode-se considerar ainda a existência de certa complementaridade entre eles. Apesar de se encontrarem em patamares diferentes e, em muitos casos, de terem necessidades diferentes, parcerias visando à capacitação mútua poderiam facilitar o processo de capacitação do sistema publico de laboratórios como um todo.

\section{Artigo recebido em 05.07.2007. Aprovado em 22.12.2007.}

\section{Notas}

\footnotetext{
${ }^{1}$ Doenças que, apesar de atingirem anualmente milhões de pessoas, não têm tratamentos eficazes. Por serem características da população mais pobre, os tratamentos apresentam baixo retorno financeiro, e não interessam ao mercado privado. Encontram-se naturalmente em regiões pobres e subdesenvolvidas.
} 
${ }^{2}$ No caso das siderúrgicas, as funções definidas por Figueiredo (2003b) foram: investimentos, processos e organização da produção, produtos e equipamentos.

\section{ReferênCias Bibliográficas}

Alves, F.,

Bomtempo, J. V., \&

Coutinho, P. (2005).

Competências para inovar na indústria petroquímica brasileira. Revista Brasileira de Inovação, 4(2), 301-327.

Associação dos Laboratórios Oficiais do Brasil. (2005).

Personalidade jurídica dos laboratórios oficias que compõem a ALFOB. Recuperado em 25 abril, 2005 , de http://www.alfob.gov.br/ perjuridica.htm

Bell, M., \&

Pavitt, K. (1995).

The development of technological capabilities. In I. U. Haque (Ed.). Trade, technology and international competitiveness. Washington: The World Bank.

Cohen, W. M., \&

Levinthal, D. A. (1990).

Absorptive capacity - a new perspective on learning and innovation. Administrative Science Quarterly, 35(1), 128-152.

Figueiredo, P. N. (2000).

Trajetórias de acumulação de competências tecnológicas e os processos subjacentes de aprendizagem: revisando estudos empíricos. Revista de Administração Pública, 34(1), 7-33.
Figueiredo, P. N. (2003a).

Aprendizagem tecnológica $e$ performance competitiva. (L. A. Monjardim, Trad.) Rio de Janeiro: Editora Fundação Getúlio Vargas. (Obra original publicada em 2001).

Figueiredo, P. N. (2003b).

Learning, capability accumulation and firms differences: evidence from latecomer steel. Industrial and Corporate Change, 12(3), 607-643.

Figueiredo, P. N., \&

Ariffin, N. (2003, setembro)

Internationalization of innovative capabilities: counter-evidence from the electronics industry in Malaysia and Brazil. Anais do Encontro Nacional da Associação Nacional de Pós-Graduação e Pesquisa em Administração, Atibaia, SP, Brasil, 27.

Kim, L. (1999).

Building technological capability for industrialization: analytical frameworks and Korea 's experience. Industrial and Corporate Change, 8(1), 111-136.

Lall, S. (1992).

Technological capabilities and industrialization. World Development, 20(2), 165-186. 
Nonaka, I., \&

Takeuchi, H. (1997).

Criação do conhecimento na empresa: como as empresas japonesas geram a dinâmica da inovação (A. B. Rodrigues \& P. M. Celeste, Trads.). Rio de Janeiro: Campus. (Obra original publicada em 1995).
Resolução RDC $n^{\circ} 210$ de 04 de agosto de 2003 (2003).

Altera a lei 6360 de 23 de setembro de 1976. Dispõe sobre vigilância sanitária, boas práticas de fabricação, dispõe sobre a utilização de nomes genéricos em produtos farmacêuticos e dá outras providências. Agência Nacional de Vigilância Sanitária. Brasília, DF. Recuperado em 20 novembro, 2004, de http:// www.anvisa.gov.br/legis/resol/rdc/ 210_03.pdf 